\title{
Performance of fibre-reinforced high-strength concrete with steel sandwich composite panels under static loading
}

\author{
C. Abraham \& K. C. G. Ong \\ National University of Singapore, Singapore
}

\begin{abstract}
This study investigates the performance of steel-concrete composite panels, which consist of fibre-reinforced high-strength concrete (FRHSC) on the compression face and a specially configured steel sandwich as tension reinforcement. The performance of the composite panel is compared to similar ordinary reinforced concrete panels. The latter is reinforced with the maximum reinforcement ratio specified by the codes. The static response of the composite panel is obtained numerically using finite element modelling and experimentally under third point bending tests. A bending capacity calculation based on the EC2 code is also carried out to predict the peak load of the composite panels. Parametric studies are done by varying the steel sandwich core design as well as the concrete layer strength. Test results showed that the EASP2 configuration with an average reduction of $33 \%$ in overall weight outperforms the high-strength concrete panels reinforced with a $3.9 \%$ reinforcement ratio. Overall, the EASP could achieve higher ductility compared to reinforced concrete panels. The numerical and analytical calculations show good accuracy in comparison to the experimental results. The combination of FRHSC and steel sandwich structure demonstrated good potential as structural replacements of reinforced concrete slabs due to better weight-to-performance ratios and high ductility properties of the panel. This study forms the first part of an ongoing study of suitable composite panels for use in resisting blasts and penetration.
\end{abstract}

Keywords: steel-concrete composite, static loading, fibre-reinforced highstrength concrete, steel sandwich. 


\section{Introduction}

Composite sandwich panels such as the metal air sandwich or cellular sandwich panels are typical composites used in critical lightweight structures. This composite paneling system is made using a structured metal core sandwiched between two metal plates that maximize build volume with minimal use of material. High stiffness, low weight ratios that yield a better weight-toperformance ratio are the main benefits of utilizing the cellular sandwich panels as structural components. Many studies were reported and concur that this type of composite panels could deliver better performance when subjected to static and dynamic loads when compared, based on total panel weight, to ordinary solid concrete or steel panels $[1,2]$.

On the other hand, steel-concrete-steel (SCS) as panels with a concrete core encased between two solid steel plates has increasing applications, from tunnel and roadway slabs to the protective structures and high performance barrier due to several advantages offered by this system. Liew and Wang [5] studied static and dynamic performance of a novel SCS panel using J-hook connectors. It was found by Sohel and Liew [3] in the static performance study of lightweight SCS slabs that the J-hook connectors can efficiently ensure composite action, especially the transfer of interface shear as well as vertical shear. In their study, SCS sandwich slabs using lightweight aggregate concrete (LWAC) were tested, loaded by centrally applied patch loads.

The concrete material also has a significant contribution in determining the panel's performance. Previous studies concluded that high-strength concrete (HSC) is too brittle to achieve its full strength potential $[4,5]$. Incorporation of fibres is a good way to overcome the brittleness of plain HSC. Fibre-reinforced concrete may be defined as composites of concrete with the incorporation of discrete, discontinuous fibres. In ordinary concrete, strength will decrease abruptly when initial cracking occurs. The weakest link is usually at the interfacial transition zone (ITZ) or at the interface between the aggregate and the cement matrix [6]. By introducing fibres into the mixture, such cracks may be bridged, limiting further propagation, leading to higher post crack ductility and greater residual capacity. These properties may yield more advantages in a composite system especially because of the fact that the tensile strength of concrete is the weakest link in most steel-concrete composite systems. In this study, $0.5 \%$ volumetric ratio of $2000 \mathrm{MPa}$ tensile strength, $13 \mathrm{~mm}$ length and $0.16 \mathrm{~mm}$ diameter straight steel fibre was added to the $\mathrm{C} 110 \mathrm{~F}$ concrete.

The composite panel in this study combines a cellular steel sandwich panel with fibre-reinforced high-strength concrete (FRHSC) as an energy absorption sandwich panel (EASP) intended for use as sacrificial cladding panels to resist blast and impact loading. The cellular steel sandwich panel as a distal layer resists most of the tension force and provides the necessary energy dissipation mechanism through plastic buckling of the core structure. The high ductility-high strength concrete will be utilized as the incident layer that receives the blast shock and imparts high stiffness properties to the composite panel. Two core structures were studied; Type 1 comprising a relatively flexible core and Type 2 , a relatively 
stiffer core. The composite panel is fabricated using a $50 \mathrm{~mm}$ thick cellular steel sandwich panel and a $60 \mathrm{~mm}$ thick FRHSC with protruding steel plates welded to the interfaces of the double layers steel plates (Figure 1). The advantage of the system are that the steel sandwich construction could be fabricated easily using laser cut machinery and ordinary welding equipment, or could be directly welded to reduce the cost.

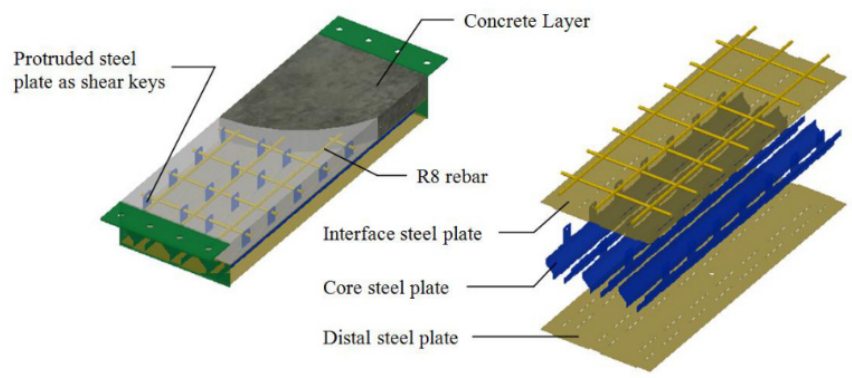

Figure 1: Proposed concrete-cellular steel sandwich composite panel.

This paper presents the first part of a three phase study of static, impact and blast performance of EASP composite panel. The panel was designed to withstand dynamic loads with better performance in total deflection compared to ordinary RC panels. The core design of Type 1 of the EASP were similar to those reported by Guruprasad and Mukherjee [7]. They carried out experimental and numerical analyses on the behaviour of layered steel sandwich panels subjected to blast loading and found that the impulse transfer was reduced substantially at the base of the cladding. The imparted energy was absorbed through core steel plastic deformation. The results suggest that such steel sandwich panels may be very efficient in dissipating blasts. Besides the dynamic performance, the static performance of the composite panel was also studied with the intention of making the panels suitable for applications as slab and wall elements.
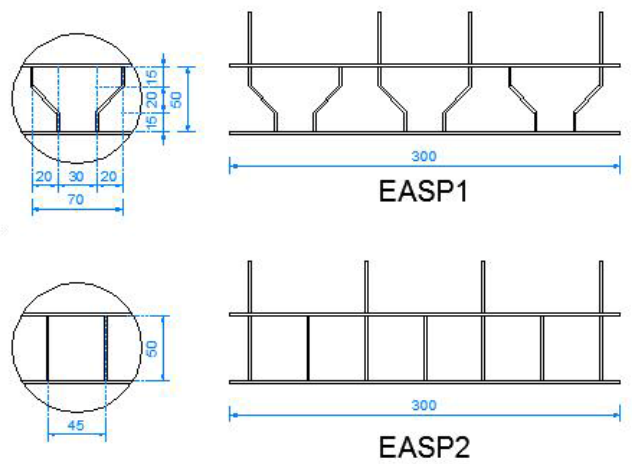

Figure 2: Section of steel sandwich core design (dimension in $\mathrm{mm}$ ). 
This paper deals mainly with the static performance of EASP comprised of concrete and cellular steel sandwich panels in comparison with RC panels with a reinforcement ratio of $3.9 \%$. Experimental and numerical study were carried out to obtain the peak strength and ductility properties from load-displacement curves. Analytical calculations were also performed to obtain the theoretical maximum load that the panel can resist.

\section{Experimental program}

The EASP fabrication begins with the steel sandwich assembly to be used as formwork to cast the concrete incident layer. The $2 \mathrm{~mm}$ thick steel plates were perforated by a laser cutting machine and welded using the manual metal inert gas (MIG) welding process. Slot joints were designed to facilitate assembly of the top and bottom steel plates of the sandwich by sets of stiffeners that also function as the core of the sandwich structure. Some of the core steel plates were designed to protrude to provide shear links between the steel sandwich structure and incident concrete layer. $5 \mathrm{~mm}$ thick steel plate was then welded to the sides of the steel sandwich panel to add end rigidity. After that, R8 rebar were inserted into the holes of the protruding steel plates. Concrete layer was then cast and cured under plastic sheets for 28 days. Three cubic concrete blocks were also cast to obtain the concrete characteristic compressive strength, $\mathrm{f}^{\prime} \mathrm{c}$.

The RC panels were also fabricated to compare their performance against proposed EASP. The reinforcement was 3T13 and 3T20 as compression and tension longitudinal rebar respectively, with $\mathrm{R} 8$ shear links (to provide in total, $3.9 \%$ reinforcement ratio). The tension rebar is calculated to have equal tension capacity as the steel sandwich layer of the EASP based on the steel area and tensile strength. The weight of the EASP is 33\% lower than that of the RC panels due to the presence of the steel sandwich in place of reinforced concrete.

The parameters of the specimens include two types of steel sandwich core design (Type 1 and 2) and three concrete strengths of NSC $60 \mathrm{MPa}$, HSC $110 \mathrm{MPa}$ and FRHSC $110 \mathrm{MPa}$. Reinforced concrete panels were also cast using HSC 110 $\mathrm{MPa}$ and FRHSC $110 \mathrm{MPa}$ for testing in this study. Hence, six EASP specimens with dimensions of $800 \times 300 \times 114 \mathrm{~mm}$ and two RC panels of the same dimensions were fabricated for static testing.

The experimental setup of the static tests utilizes a three point bending test configuration. The end supports were pin and roller steel rockers. A line load with displacement control mode was applied at a loading rate of $0.1 \mathrm{~mm} / \mathrm{min}$. The loaded area of the steel rocker is $100 \times 300 \mathrm{~mm}$. One load cell below the hydraulic actuator and two displacement transducers were used to measure the load, interface steel plate displacement and distal steel plate displacement respectively. For the EASP specimens, four strain gauges each were installed on the interface and distal steel plates. The concrete was painted white using limewater mixture to aid in visually inspecting for cracks during the test. 

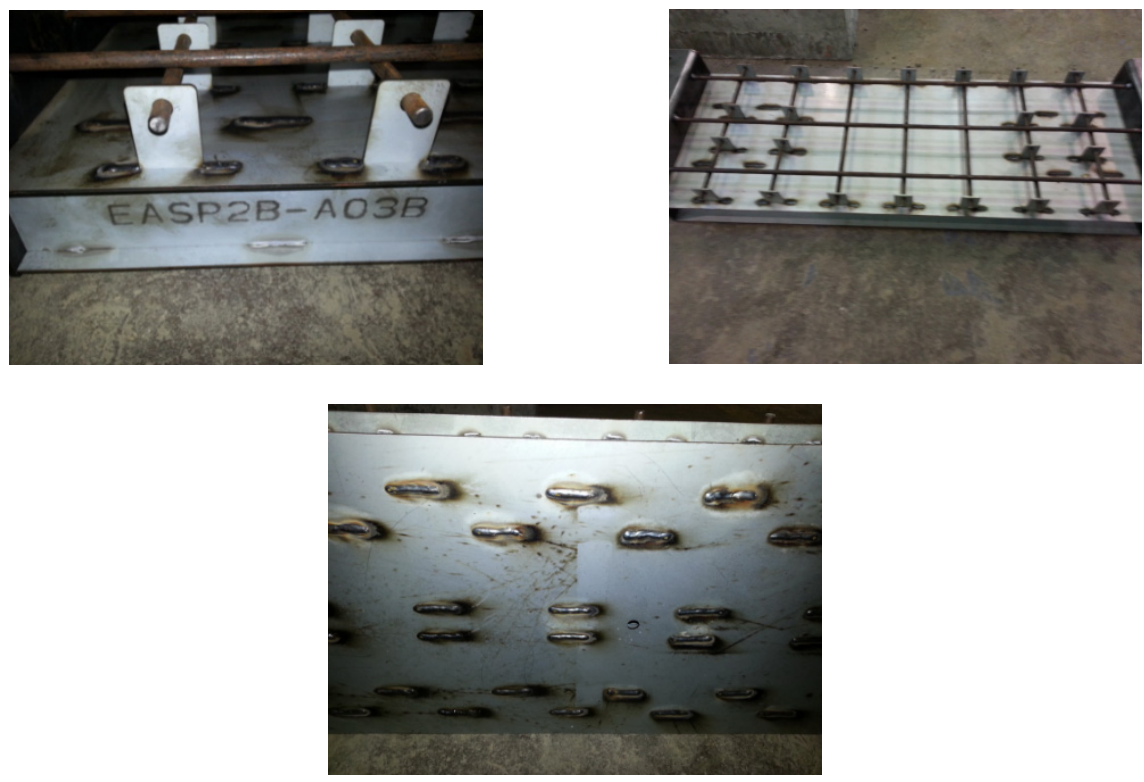

Figure 3: Steel sandwich fabrication.

\section{Numerical study}

Finite element program LS-DYNA implicit software was used to simulate quasistatic loading on the composite and reinforced concrete panels. Kong et al. [8] studied axially restrained non-composite SCS panels under static and impact loadings. The experimental results were compared to those from the numerical models using LS-DYNA software. It was found that the results demonstrated reasonable correlation with the test results. It may indicate that the software has good accuracy for static simulation application.

The models for analyses were developed in LS-Prepost software with proper meshing size chosen from a convergence study. The concrete was modelled using rectangular 8-node solid constant stress elements with $5 \mathrm{~mm}$ mesh size and Hourglass Type 6, rebar as truss beam elements and steel plates as thick shell elements. The interface between the concrete and steel element were idealized with full connectivity between common nodes. Langrangian Implicit method was used with automatic time step adjustment. IRATE in IMPLICIT_DYNAMICS was turned off to remove strain rate effects from the material model.

The concrete material properties are presented in Table 1. Karagozian and Case (K\&C) Concrete Model Release III (MAT72R3) was adopted to model the concrete constitutive behaviour. The $\mathrm{K} \& \mathrm{C}$ model is proven to be a suitable material model for quasi-static, blast and impact loads [9]. The model features automatic parameter generation that was used by inputting concrete mass density (RO), Poisson's ratio (PR), unconfined compression strength in negative format (A0), and values of RSIZE and UCF according to the units used. 
Table 1: Concrete properties.

\begin{tabular}{llll}
\hline Properties & FRHSC & HSC & NSC \\
\hline Density $\left(\mathrm{kg} / \mathrm{m}^{3}\right)$ & 2480 & 2450 & 2400 \\
f'c $^{\prime}(\mathrm{MPa})$ & 124 & 105.8 & 66 \\
Young's modulus $(\mathrm{GPa})$ & 51.6 & 48.7 & 36.4 \\
Poisson ratio & 0.2 & 0.2 & 0.2 \\
\hline
\end{tabular}

The calibration method of the K\&C model involves the alteration of $b_{1}$ and $b_{2}$ parameters that are directly correlated to the compression and tension fracture energy of the concrete. Single element tests in accordance with the mesh size used was first simulated with the default parameters. The area under the post-peak portion of the stress displacement curve was then computed and compared to the experimental stress-strain curve. The calibration involves several iterations to equalize the two areas and once completed became the custom input of the material data used in modelling.

MAT3 Plastic Kinematic was used for steel material based on coupon and rebar tension test results. The steel properties detail is showed on Table 2. Strain rate effect for both steel and concrete are turned off to eliminate the strength increase.

Table 2: Steel material properties.

\begin{tabular}{llll}
\hline \multirow{2}{*}{ Steel } & $\begin{array}{l}\sigma_{\mathrm{y}} \\
\mathrm{MPa}\end{array}$ & $\begin{array}{l}\sigma_{\mathrm{u}} \\
\mathrm{MPa}\end{array}$ & $\begin{array}{l}\mathrm{E}_{\mathrm{tan}} \\
\mathrm{GPa}\end{array}$ \\
\hline $\mathrm{T} 20$ & 495 & 596 & 1.755 \\
$\mathrm{~T} 13$ & 562 & 665 & 1.610 \\
$\mathrm{R} 8$ & 495 & 596 & 3.765 \\
2 mm plate & 244 & 316 & 0.965 \\
\hline
\end{tabular}

The load was recorded in the numerical simulation using DATABASE and ASCII output keywords. The FE model can be seen from Figure 4 below.

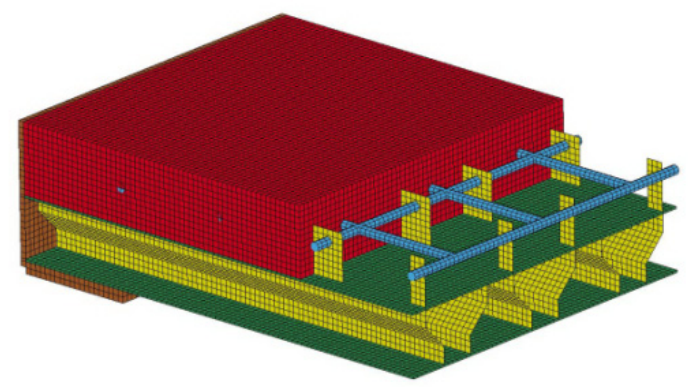

Figure 4: EASP1 FE model. 


\section{Results and discussion}

The experimental results are presented in the form of load vs. displacement graphs, Figure 5 and 6.

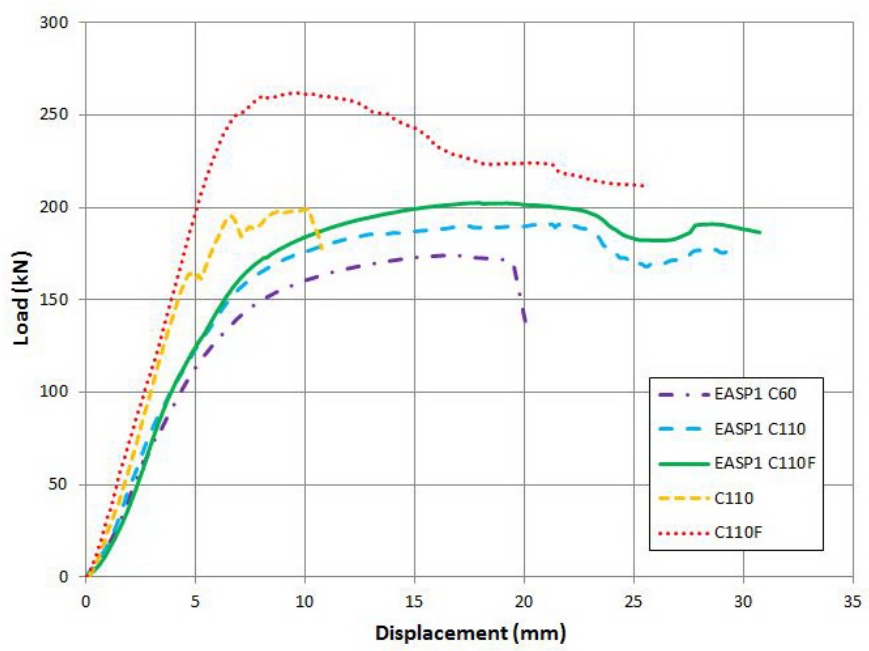

Figure 5: EASP 1 vs. RC load-displacement history.

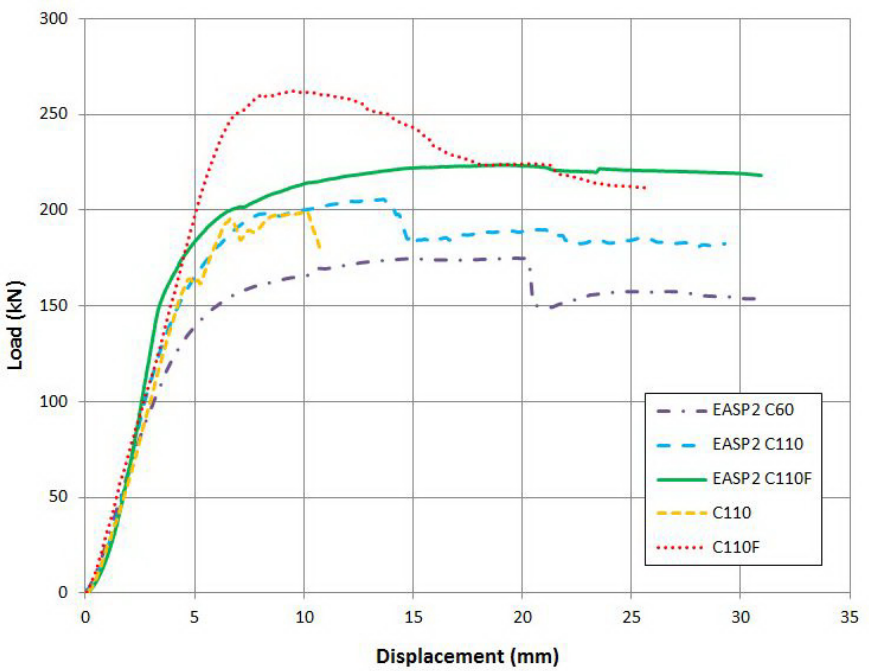

Figure 6: EASP 2 vs. RC load-displacement history. 
Figure 5 shows the load-displacement curve of the EASP1 panel with various concrete types and the RC panel cast using the HSC and FRHSC materials. When comparing EASP with the RC panel, it can be seen from the graph that EASP1 has lower peak strength compared to the RC panel cast with a similar concrete type. This might be caused by the flexibility of core type 1 . The core is designed to be flexed when subjected to dynamic impact or blast loading. As a result, the lower stiffness resulted in lower peak strength and tangent modulus. The RC panel with fibre reinforced concrete could achieve the best performance due to the high ductility-high strength properties of the concrete. However, the RC panel without fibre yields much lower peak strength because of the brittleness of HSC. The presence of fibres in the concrete gave substantial benefits in the form of higher peak strength and ductility.

Figure 6 shows the experimental load vs. deflection curve of the EASP2 and the RC panel for comparison. The EASP2 with HSC material has higher peak strength and ductility than the RC panel cast using the same concrete. The Type 2 sandwich core could provide better stiffness that yielded higher elastic modulus and higher peak strength than Type 1 core sandwich. The EASP also exhibited high ductility properties that can sustain large deformation. EASP2 with FRHSC material could withstand substantial displacement with the moment resistance constant. This may indicate that the EASP is suitable for high deformation applications such as blast protective panels.

The numerical simulation results are plotted in Figures 7 and 8.

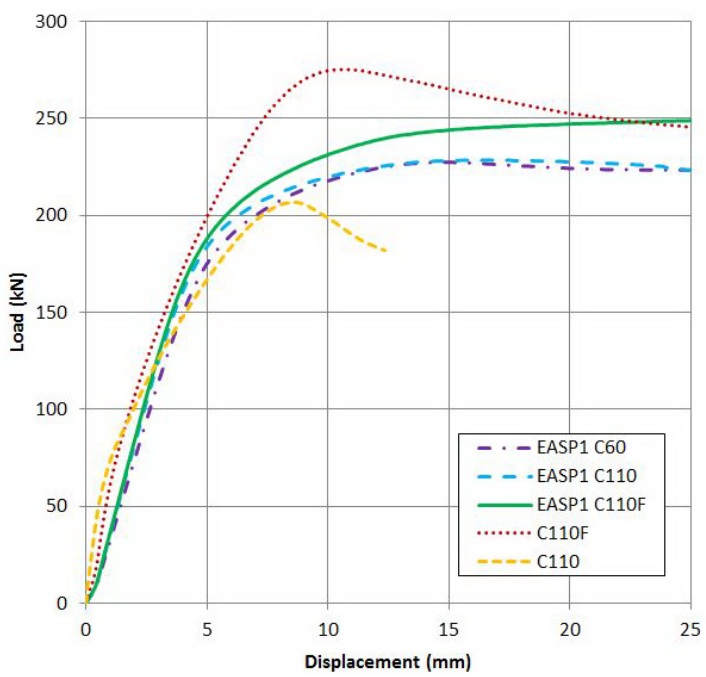

Figure 7: Numerical load-displacement history (EASP1 vs. RC). 


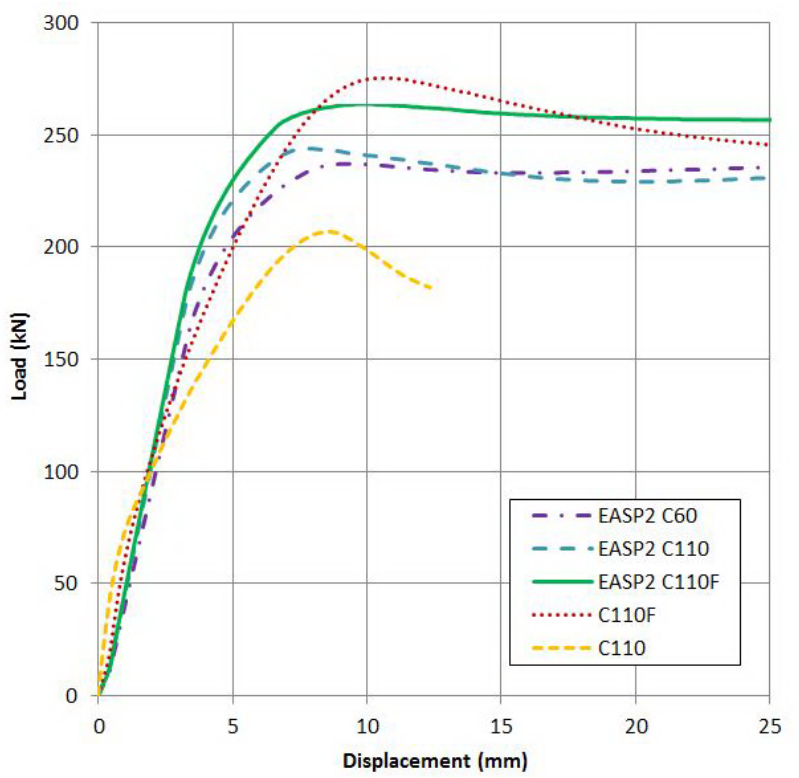

Figure 8: Numerical load-displacement history (EASP2 vs. RC).

From experimental and numerical results of the EASP, it could be seen that the overall strength values of the FE simulation are higher than those of the static experimental tests. This could be attributed to the bond-slip performance at the steel-concrete interface observed experimentally. The numerical simulation did not take this into account, and perfect bond was assumed between the two materials. Although the protruding steel plates are used as shear connectors, the shear transfer only occurs within the limited region of the connecting plate and the flexibility of the steel material could result in localized loss of stiffness. If the bond strength can be calibrated, then spring elements linking the two nodes at the steelconcrete interface could be utilized to achieve greater simulation accuracy. However, due to limited time and experimental constraints this will be carried out in the future.

The ultimate moment capacity of the panel based on EC2 code was also calculated to compare the analytical peak strength with the experimental results. The values of the analytical calculations are summarized in Table 3 . It can be seen that the peak load of EASP seem to match the experimental values, even outperform the theoretical maximum load for EASP2. The C110F RC panel calculation also yields accurate value; however, the $\mathrm{C} 110$ (plain high strength concrete) RC panel exhibit much lower strength than the theoretical one. This may show the brittle weakness of HSC and proves the effectiveness of the fibre in helping the concrete achieve its potential strength. 
Table 3: Panel peak load comparison.

\begin{tabular}{lcccrr}
\hline \multirow{2}{*}{ Specimen } & \multicolumn{3}{c}{ Maximum load (kN) } & $\begin{array}{c}\text { E/N } \\
\text { (\%) }\end{array}$ & $\begin{array}{l}\text { E/A } \\
(\%)\end{array}$ \\
\hline EASP1 C60 & 174.3 & 227.4 & 173.7 & -23 & 0 \\
EASP1 C110 & 190.9 & 228.4 & 198.6 & -16 & -4 \\
EASP1 C110F & 202.6 & 250.4 & 201.9 & -19 & 0 \\
EASP2 C60 & 174.8 & 237.8 & 173.7 & -26 & 1 \\
EASP2 C110 & 205.6 & 243.8 & 198.6 & -16 & 4 \\
EASP2 C110F & 223.5 & 263.6 & 201.9 & -15 & 11 \\
C110 & 199.1 & 206.0 & 254.1 & -3 & -22 \\
C110F & 262.2 & 275.2 & 268.1 & -5 & -2 \\
\hline
\end{tabular}

\section{Conclusions}

In this paper, the static performance of EASP composite panels was investigated and compared to RC panels of the same geometric dimensions and tension capacity. Parametric study was carried out, utilizing two types of the core structure and three types of concrete material. It was found that the EASP2 with HSC material outperform the RC panels in terms of peak strength and ductility. EASP2 with FRHSC materials could perform similar to the C110F RC panels based on results of the FE simulation. However, the slip and flexibility of the shear joint seems to result in loss of stiffness and leads to much lower peak strength. Overall, all EASP panels could achieve much higher ductility due to the cellular core of the steel sandwich as distal layer.

Numerical simulations were carried out to assess the EASP and RC panel performance using implicit method. The results are in agreement with the experimental data with reasonable accuracy in the peak strength values. The RC FE model could reach more than $90 \%$ accuracy in the peak strength estimation whereas EASP model could yield accuracies greater than $74 \%$. In general, the model can accurately emulate the behaviour (displacement and failure mode) of the panels subjected to static loading.

\section{References}

[1] Rathbun, H.J., et al., Performance of metallic honeycomb-core sandwich beams under shock loading. International Journal of Solids and Structures, 2006. 43(6): pp. 1746-1763.

[2] Radford, D.D., et al., The response of clamped sandwich plates with metallic foam cores to simulated blast loading. International Journal of Solids and Structures, 2006. 43(7-8): pp. 2243-2259.

[3] Sohel, K.M.A. and J.Y. Richard Liew, Steel-Concrete-Steel sandwich slabs with lightweight core - Static performance. Engineering Structures, 2011. 33(3): pp. 981-992. 
[4] Magnusson, J., M. Hallgren, and A. Ansell, Air-blast-loaded, high-strength concrete beams. Part I: Experimental investigation. Magazine of Concrete Research, 2010. 62(2): pp. 127-136.

[5] Liew, J.Y.R. and T.Y. Wang, Novel Steel-Concrete-Steel Sandwich Composite Plates Subject to Impact and Blast Load. Advances in Structural Engineering, 2011. 14(4): pp. 673-687.

[6] Zampini, D., H.M. Jennings, and S.P. Shah, The interfacial transition zone and its influence on the fracture behavior of concrete. Microstructure of Cement-Based Systems/Bonding and Interfaces in Cementitious Materials, 1995. 370: pp. 357-366.

[7] Guruprasad, S. and A. Mukherjee, Layered sacrificial claddings under blast loading Part II - experimental studies. International Journal of Impact Engineering, 2000. 24(9): pp. 975-984.

[8] Kong, S., A. Remennikov, and B. Uy, Numerical simulation of highperformance SCS panels under static and impact loading conditions, in Incorporating Sustainable Practice in Mechanics and Structures of Materials 2010, CRC Press. pp. 591-596.

[9] Wu, Y., J.E. Crawford, and J.M. Magallanes, Performance of LS-DYNA Concrete Constitutive Models, in 12th International LS-DYNA Users Conference 2012: Michigan, USA.

[10] Nie, J. and C. Cai, Steel-Concrete Composite Beams Considering Shear Slip Effects. Journal of Structural Engineering, 2003. 129(4): pp. 495-506. 